\title{
Validity and reliability study of the vaccine hesitancy scale in Turkish sample
}

\author{
Hacer Yalnız Dilcen ${ }^{1} \odot$, İlknur Dolu²๑, Zeynep Turhan ${ }^{3} \odot$ \\ ${ }^{1}$ Department of Midwifery, Bartın University, Faculty of Health Sciences, Bartın, Turkey \\ ${ }^{2}$ Department of Nursing, Bartın University, Faculty of Health Sciences, Bartın, Turkey \\ ${ }^{3}$ Department of Social Work, Bartın University, Faculty of Health Sciences, Bartın, Turkey
}

\begin{abstract}
Objectives: This study aims to test the validity and reliability of the Vaccine Hesitancy Scale (VHS) developed by Larson for Turkish society due to the limited measures of vaccine hesitancy studies in Turkey. This scale can help advance research and vaccination policy for human well-being.

Methods: Two hundred fourty-six participants completed the first questionnaire in Turkish between September and October 2020. The sample of the study was determined to consider the number of items on the 9-item scale is more than 27 times. The retest was used to test the validity of the scale in the study.

Results: The ratio of the scale to chi-square degrees of freedom is 2.29. This can be considered as a sufficient fit. As a result of the first level Confirmatory Factor Analysis (CFA), the values of the goodness of fit suggested that the two-factor model can be considered compatible with the data. The Cronbach's alpha of the total items of the scale is $=.801$. Factor 1 which was the 'lack of confidence' of Cronbach's alpha was 0.904. Factor 2 that was the 'risks' was 0.742 . The reliability and validity of the VHS analysis revealed a two-factor structure with construct and criterion validity to detect vaccine hesitancy.
\end{abstract}

Conclusions: VHS is recommended to be used as a data collection tool in health care services to detect the level of vaccine hesitancy among the public. The adaptation of VHS into the Turkish language can help health care providers and immunization policy makers to improve effective approaches by focusing on the individuals' confidence in vaccination.

Keywords: Confidence in vaccination, vaccine hesitancy, scale, vaccine

V accine immunization is undoubtedly the best cost-effective method of combating preventable diseases of the last century [1]. The vaccine provides both individual and social immunization [2]. Vaccination programs are the most effective method that has been used to prevent and eliminate communicable diseases and reduce morbidity and mortality rates for public health [1]. According to the report of the WHO (2019); global immunization prevents 2-3 million deaths in a year. The global immunization rate has been around $85 \%$ for the last few years. It is stated that the vaccination can prevent 1.5 million more people from deaths per year by increasing the immunization rates to the targeted level [3].

When the vaccination was first introduced in Europe in the 18th century to prevent smallpox, people had started to hold hesitancy about vaccination [4-6]. "Vaccination hesitant" mean delayed acceptance or re- 
fusal of vaccination despite the availability of services [7]. Vaccine hesitancy was defined as attitudes such as anxiety or doubts as well as a behavior [8]. Factors contributing to vaccine hesitancy are different based on the specific vaccine, individual factors, social influences, and environmental conditions [9, 10]. For example, there are different attitudes around vaccine hesitancy related to the distrusting vaccines or healthcare providers and different perceptions of the need for vaccination $[11,12]$.

WHO (2019) identified vaccine hesitancy as an important issue that can solve ten global health problems. Importantly, it plans to resolve in recognizing the importance of community immunization and the increase in vaccine-preventable diseases. WHO has proposed a multifaced strategy to reduce and stop individuals' vaccine hesitancy by developing vaccination programs to proactively prevent vaccine hesitations in many countries [3]. In the last 20 years, especially in the last 8 years, concerns against vaccination and vaccine hesitancy have started to increase again, and WHO established the "Vaccine Hesitancy Working Group" in 2012 and began to investigate the reasons for vaccine rejection [13]. This research group developed a measure to explore vaccine hesitancy through a systematic review of existing research, a review of questions used by the WHO and UNICEF Joint Reporting Form, and expert consultation [14]. WHO recommended to implementing these tools to evaluate and research in different contexts to determine whether they can be used as a basis for measuring vaccine hesitancy. Moreover, they focused on how this can be adapted to low- and middle-income countries [13]. In response to this recommendation, one of these tools with the potential to measure and compare vaccine hesitancy across countries and overtime was the Vaccine Hesitancy Scale (VHS). This tool has been approved and evaluated in Canada, Guatemala, and more recently in the United Kingdom and Sudan [1518]. Findings from these four countries revealed that VHS is a valid and reliable tool for measuring vaccine hesitancy. Also, it was recommended that VHS needs further adaptation and validation in different contexts [15-18]. The development and standardization of a vaccine hesitancy measure are crucial to improving jurisdiction and the ability to measure, evaluate and compare over time.

The development and standardization of a vaccine hesitancy measure are key to improve jurisdiction and the ability to measure, evaluate and compare over time. Recently, several scholars also paid attention to the COVID-19 vaccine hesitancy as a public health problem [19-23]. Therefore, the VHS can be used to examine the level of vaccine hesitancy during the COVID-19 crisis. Importantly, there are no existing tools for measuring vaccine hesitancy in Turkey. The examination of the vaccination hesitancy has been started by using different measures at the international level. Some of these measures are the 8-item Vaccine Confidence Scale [24], 18-item Parental Attitudes towards Childhood Vaccination Scale [25], 7-Item Vaccination Conspiracy Belief Scale [26], 12-item Vaccination Attitudes Review Scale [27], 5-item Vaccine Attitude Scale [28]. Larson et al. [14] tried to standardize the vaccine hesitancy measure. These studies help us to recognize the importance of examining the vaccine hesitancy to improve confidence in vaccination. Insufficient quality of the data prevented the monitoring of various indicators. While vaccine hesitancy is an important public health problem, the measures around vaccine hesitancy have been limited to cover the complexity of confidence in the vaccination process [3]. A standardized, validated measurement tool of vaccine hesitancy will help develop the research and immunization policy. This tool has the potential to widely use to explore the relationship between vaccine instability, vaccine hesitancy and vaccine coverage, compare vaccine hesitancy between countries. Also, it can evaluate how individuals' vaccine hesitancy can change over time. This article aims to adapt the Vaccine Hesitancy Scale developed by Larson et al. [14] to Turkish society and examine whether it is a valid and reliable measurement tool in the adult sample.

\section{METHODS}

Considering the lack of reliable and valid measures around vaccine hesitancy in Turkey, this article tested the validity and reliability of the VHS in the Turkish language. The VHS was developed by Larson et al. [14] and tested the validity and reliability by Shapiro et al. [16]. This study examined the structure and internal consistency of the scale, construct validity, and criterion validity. We used an online survey to 
test the reliability and validity of VHS in Turkish language. The participants who were recruited through social media (e.g., Instagram, Facebook, WhatsApp, Twitter etc.) lived in Turkey. The data were collected through applications on the web between October and November 2020. The inclusion criteria were (i) accepting to participate in the research, (ii) being over 18 years old, and (iii) being able to read and write. Data were collected by random sampling method. The sample of the study was more than 27 times the number of items on the 9-item scale, and 246 participants completed the first questionnaire. It is also stated that the sample size should be five or even ten times the number of observed variables [29, 30]. For the calculation of the reliability coefficients, the Re-test method, which is the application of a measurement tool to the same subject group twice in the same conditions and at a certain time interval, was used [29, 30]. Fifteen days after the first questionnaire, the same questionnaire was sent to the participants again for retest. Seventy-two participants completed the re-test part. Research data were collected by using the Participant Information Form and the VHS.

\section{Ethical Considerations}

Bartın University Ethics Committee Approval was obtained to collect research data (2020-SBB-0204$22 / 08.10 .2020)$. The first question of the online form was about consent regarding whether or not the person agreed to participate in the study. In this way, participants read the information about the study and give consent for participation in the study. Participants who gave their consent were included in the study.

\section{Data Collection Tools}

\section{Participant Information Form}

The authors prepared this form to explore the participants' socio-demographics. These socio-demographic items were about age, educational status, marital status, the number of children, etc.

\section{Vaccine Hesitation Scale}

This scale was developed by Larson et al. [14] to examine vaccine hesitancy, attitudes and problems regarding vaccination. Shapiro et al. [16] found that VHS has two factors with construct and criterion validity in determining parents who are hesitant about vaccination. In this study, Shapiro et al. [16] tried to verify the nine-item VHS Likert-type scale question that was validated. The scale consists of 9 questions and two sub-dimensions: lack of confidence and risks. Items $1,2,3,4,5,6,7$ are included in the lack of confidence dimension of the scale and items 8, 9 are included in the dimension of the risks. Scale questions are reverse coded up to 1-7. The scale has a five-point Likert-type rating included five points: "1-strongly disagree; 2-disagree; 3-neither agree nor disagree; 4agree; "5-strongly agree". As the seven items were reversed, the higher score indicates more vaccine hesitancy. Scores range from 9 to 45 for the total number of sub-groups. The Cronbach alpha coefficient for the "lack of confidence" sub-dimension of the original scale was 0.90 and the "risks" was 0.64.

\section{Statistical Analysis}

Frequency, standard deviation, and item mean were performed to assess the data revealed from the VHS. Pearson correlation analysis for item analysis was used to test the reliability. Cronbach's alpha analysis for internal consistency of the total scale and its sub-dimensions was implemented. Also, test-retest scores for time invariance were compared. SPSS version 22 and Amos version 24 programs were used for the analysis.

\section{RESULTS}

\section{Sample Characteristics}

The sample consisted of $62.2 \%$ female, $37.8 \%$ male participants. Their educational status was 52.8\% undergraduate, $17.5 \%$ primary education, $15.4 \%$ graduate and $14.2 \%$ high school level. The average age of the participants was $36.47 \pm 37.43$ years, and the average monthly income of the family was $6.056 .36 \pm$ 6.946.97 Turkish Liras. It was determined that $65.4 \%$ of the participants were married and $61.4 \%$ had children. $58.9 \%$ of participants heard negative information about vaccines.

\section{The Validity of the Vaccine Hesitation Scale Translation Procedures}

Permissions of adopting the scale in the Turkish language were obtained via e-mail from Heidi J. Larson who is the original developer of the scale and Gilla K. Shapiro who psychometrically evaluated it. The 
original scale was translated from English to Turkish by three faculty members from the departments of Social Work, Psychology and Public Health, who have mastered both English and Turkish languages, are native speakers of Turkish. Three translated versions were compared by the authors and the researchers developed a common Turkish text from these three Turkish translations. The linguists compared the scale that became original. The study started after the linguists gave congruence.

\section{Content Validity}

The opinions of 20 experts were taken to evaluate the compatibility of the scale, which was translated into Turkish, with our culture and language. The profession of these experts included public health (including one professor, 3 associate professors and 4 assistant professors) and midwifery (including a professor, 3 associate professors and 4 assistant professors). However, no feedback was received from four faculty members. The expert team was informed about the study process and requested to evaluate 9 questions regarding the suitability of the question contents, the status of meeting the area to be measured, the scope and language validity.

Based on the technique developed by Lawshe
[31], the qualitative data obtained following expert opinions were converted into quantitative data after calculating the SVS and SVI values to determine the content validity and language validity of the items to be included in the scale. The content validity of the study was 0.91 and the language validity SVI value was 0.80 . The fact that the SGI value obtained is greater than the SVI value (SGI > SVI) indicates that the content validity of the remaining items in the scale is statistically significant $[31,32]$. The Kaiser Meyer Olkin (KMO) value of VHS was 0.862 . The normal range of KMO value indicates that the explanatory factor analysis results can be applied to the data. As a result of the Bartlett Sphericity test, there was a significantly high correlation between variables and the data that means suitable for explanatory factor analysis (X2: 1156.115, SD: 36, $p<0.001$ ).

\section{Reliability Analyzes}

First, exploratory factor analysis (EFA) was performed in the study. According to EFA results, there are six items in the first factor and three items in the second factor. It was seen that the 5th item, which was located under the first factor in the original scale, was located under the second factor differently in the Turkish version of the scale. The eigenvalue of VHS's lack

Table 1. Distribution correlation of the items of the Vaccine Hesitation Scale by sub-dimensions

\begin{tabular}{lcc}
\hline Items & $\begin{array}{c}\text { Lack of confidence } \\
\text { sub-dimension }\end{array}$ & $\begin{array}{c}\text { Risk } \\
\text { sub-dimension }\end{array}$ \\
\hline $\begin{array}{l}\text { 1. Childhood vaccines are important for my child's health } \\
\text { 2. Getting vaccines is a good way to protect my child/children from } \\
\text { disease }\end{array}$ & 0.82 & 0.90 \\
3. Childhood vaccines are effective & 0.86 & \\
$\begin{array}{l}\text { 4. Having my child vaccinated is important for the health of others } \\
\text { in my community }\end{array}$ & 0.86 & 0.77 \\
5. All childhood vaccines offered by the government program in & & \\
my community are beneficial & & \\
6. The information I receive about vaccines from the vaccine & 0.74 \\
program is reliable and trustworthy & 0.75 & \\
7. Generally, I do what my doctor or health care provider & & 0.82 \\
recommends about vaccines for my child/children & & 0.84 \\
8. New vaccines carry more risks than older vaccines & & 1.92 \\
9. I am concerned about serious adverse effects of vaccines & 4.25 & $22.46 \%$ \\
Eigenvalue & $46.22 \%$ & 0.742 \\
\hline $\begin{array}{l}\text { Explained Variance Total (68.69\%) } \\
\text { Cronbach's alpha }\end{array}$ & 0.904 & \\
\hline
\end{tabular}


of trust sub-dimension was 4.25 and the variance was $46.22 \%$; the eigenvalue of the risk sub-dimension was 1.92 and the variance was $22.46 \%$. The total variance explained is $68.69 \%$ (Table 1). It would be possible that the items of the scale, which consists of nine items, are distributed in a balanced way. One of the positive features of the scale is that the number of items distributed among the factors is not less than three [33].

To achieve the item-total score correlation; it is recommended that the sample size should be at least 100-200 people or at least 5 people should answer for each item [34]. If the item-total score correlation coefficient is 0.30 and above, its reliability is considered to be $\operatorname{good}[34,35]$. (see Table 1). In the lack of confidence sub-dimension of the scale, the item correlation coefficient was between 0.74 and 0.90 . In the lower height of the risks, the coefficient was between $0.77-0.84$. Therefore, the reliability of the scale was found to be good.

The internal consistency coefficient was examined for the reliability of VHS. For this, the Cronbach Alpha Coefficient was first measured. Cronbach's alpha for the instrument was .801 for the total scale. Cronbach's alpha for Factor 1 was .904 and Factor 2 was 0.742 (Table 1). If the Cronbach Alpha Coeffi- cient is $0.60-0.80$, it is highly reliable, and $0.80-1.00$ indicates high reliability [36]. Lack of confidence subdimension of internal consistency coefficients of VHS was high reliability, and the risks sub-dimension were found quite reliable.

\section{Structure Validity}

CFA was performed to evaluate the validity of the one-factor structure of the VHS, which consists of two sub-dimensions and a total of 9 items in the Turkish sample group. The results obtained in the first construct validity analysis were found sufficient. The scale illustrates $68.69 \%$ of the variance with this form. The first level factorial structure of VHS (6-item lack of confidence and 3-item risk factor structure), which consists of two sub-dimensions and a total of 9 items. This is presented in Fig. 1. As a result of CFA, the goodness of fit values of the scale obtained (x2 (26, n = 246) $91.75 ; p<0.008 ; \mathrm{x} 2 / \mathrm{df}=2.290 ; \mathrm{CMIN}=$ 59.534; the root mean square error of approximation $($ RMSEA $)=0.073$; the comparative fit index $(\mathrm{CFI})=$ .97 ; the goodness of fit index $(\mathrm{GFI})=.92)$ and twofactor shows that the model can be considered compatible with the data. These results show that the data obtained from the research are compatible with the predicted institutional structure of VHS (two-factor

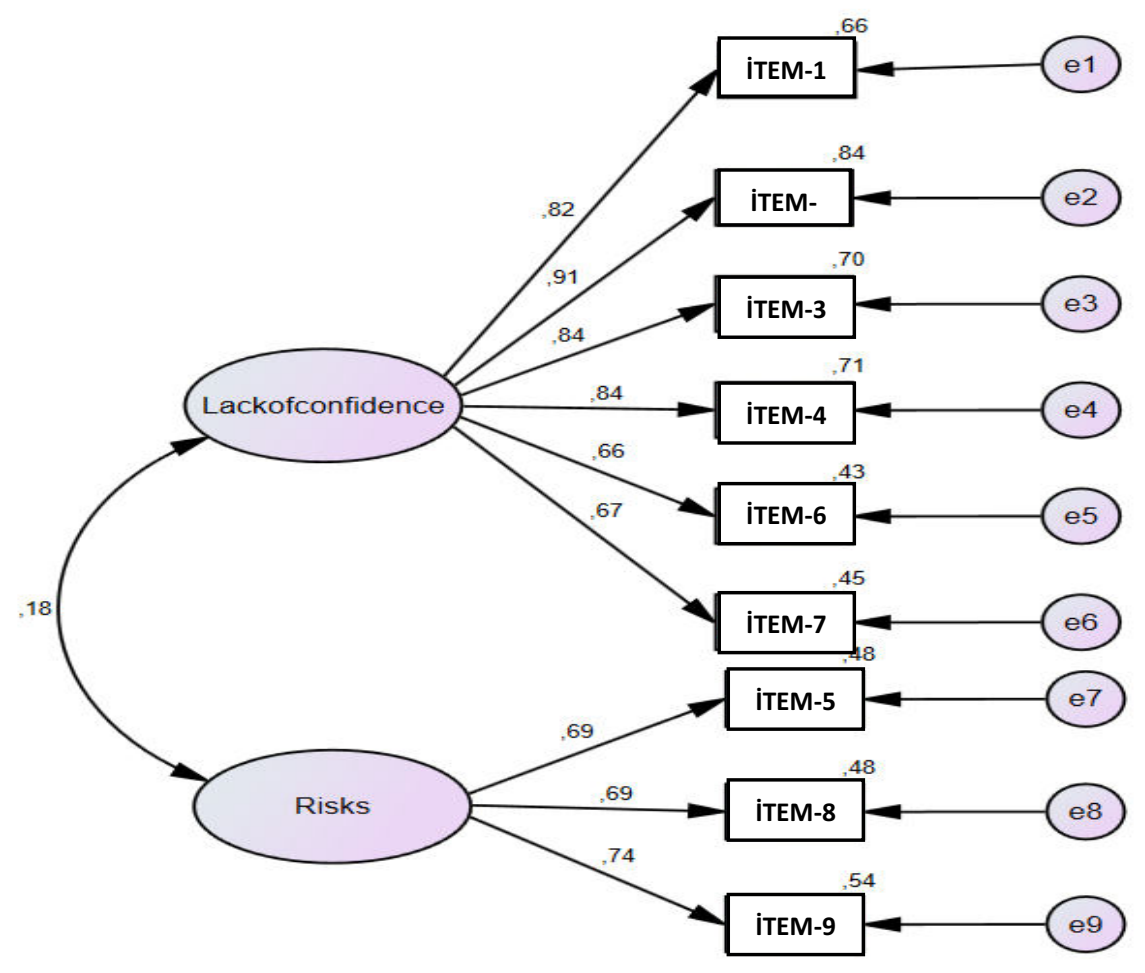

Fig. 1. First level factorial structure of the Vaccine Hesitation Scale. 
Table 2. Standard regression coefficients of the Vaccine Hesitation Scale

\begin{tabular}{|c|c|c|c|}
\hline \multirow[t]{2}{*}{ Scale items } & \multicolumn{3}{|c|}{ Standard Regression Coefficients } \\
\hline & $\begin{array}{c}\text { Lack of confidence } \\
\text { sub-dimension }\end{array}$ & Risk s & dimension \\
\hline VHS -1 & 0.82 & & \\
\hline VHS -2 & 0.91 & & \\
\hline VHS -3 & 0.84 & & \\
\hline VHS -4 & 0.84 & & \\
\hline VHS -5 & & & 69 \\
\hline VHS -6 & 0.66 & & \\
\hline VHS -7 & 0.67 & & \\
\hline VHS -8 & & & 69 \\
\hline VHS -9 & & & 74 \\
\hline Fit indices & Good fit & Acceptable fit & Scale values \\
\hline$\chi^{2 / d f}$ & $0 \leq \chi^{2} / \mathrm{sd} \leq 2$ & $2 \leq \chi^{2} / \mathrm{sd} \leq 5$ & 2.290 \\
\hline$p$ value & $0.05<\mathrm{p} \leq 1.00$ & $0.01<\mathrm{p} \leq 0.05$ & $<0.001$ \\
\hline RMSEA & $0 \leq \mathrm{RMSEA} \leq 0.05$ & $0.05 \leq \mathrm{RMSEA} \leq 1.0$ & 0.073 \\
\hline NFI & $0.95 \leq \mathrm{NFI} \leq 1.0$ & $0.90 \leq \mathrm{NFI} \leq 0.95$ & 0.949 \\
\hline CFI & $0.95 \leq \mathrm{CFI} \leq 1.0$ & $0.90 \leq \mathrm{CFI} \leq 0.95$ & 0.971 \\
\hline GFI & $0.95 \leq \mathrm{GFI} \leq 1.0$ & $0.90 \leq \mathrm{GFI} \leq 0.95$ & 0.925 \\
\hline
\end{tabular}

$\mathrm{CFI}=$ the comparative fit index, GFI $=$ the goodness of fit index, NFI $=$ the normed fit index, RMSEA $=$ the root mean square error of approximation, VHS = Vaccine Hesitation Scale

model). As seen in Table 2, the acceptable fit of $\mathrm{x}^{2} / \mathrm{df}$, RMSEA, the normed fit index (NFI) and GFI values show a good fit of CFI values. As a result of the analyzes, it can be interpreted that the original structure of the scale with two factors is compatible with Turkish culture. In the standard regression coefficient analysis of VHS items and scale sub-dimensions, "lack of confidence" was found between 0.66-0.91, and the "risks" sub-group was found 0.69-0.74. The 9-item form obtained to measure the VHS retest reliability was sent again fifteen days later and 76 people responded. Pearson's correlation coefficients were calculated and it was found that the correlations found were significant.

\section{DISCUSSION}

This study examined the psychometric properties of the "Vaccine Hesitancy Scale" that adapted to Turkish. Shapiro et al. [16] calculated the common variance of the 10 -item scale as $66.73 \%$, and the 9-item English version as 57.25. Also, Luyten et al. [37] used the English version and determined that the scale consisting of two factors and 9 items explained $71.8 \%$ of the total variance. Sabahelzain et al. [18] observed that the two-factor structure of the 10-item version of the scale made up $50.8 \%$ of the total variance in the study of adapting the scale to the Saudi language. This study was found that the scale consisting of two factors and a total of 9 items constituted $68.69 \%$ of the total variance. It is similar to the studies done with VHS.

In the confirmatory factor analysis, analyzes were performed for two factors of 9 items of the scale. It clearly showed the best fit in the analysis results. Thus, a two-factor structure consisting of a 6-item 'lack of confidence' section and a three-item 'risks' section 
showed the best psychometric properties of VHS. The study by Shapiro et al. [16] found that confirmatory factor analysis was performed for both 9 and 10-item versions of the scale and both one and two-factor solutions, and clearly showed the best fit for the two-factor 9-item version $(\mathrm{RMSEA}=0.0750, \mathrm{CFI}=0.9$, TLI $=09666)$. Similarly, we found that the 9 -item version showed the best goodness of fit. Many studies also found that CFA complies well [16, 18, 37].

Shapiro et al. [16] removed item 10, a nine-item VHS was divided into two factors, seven and two, respectively. In their study, the first factor (consisting of seven items) represents 'lack of confidence' and the second sub-dimension (consisting of two items) represents 'risks'. In the study, as a result of the explanatory factor analysis in 9 items of VHS, a two-factor structure with VHS subscales characterized by 'lack of confidence' and 'risks' was revealed. The first subdimension (consisting of six items) represents "lack of confidence" and the second sub-dimension (consisting of three items) represents "risks". Therefore, the $5^{\text {th }}$ item was distributed under the risk subgroup. CFA analysis results in this direction showed good harmony. Domek et al. [15] stated that there are five items loaded on the first factor in his study, and these are primarily related to vaccine trust and positive attitude towards vaccines. There were two items loaded into the second sub-dimension regarding vaccination risk and peace of mind, and perceptions that vaccines are not beneficial. However, items in each subscale were loaded differently from other studies, because in our study, the 'lack of confidence' subscale consisted of 6 items and the risks subscale had 3 items. Shapiro et al. [16] stated that for the future development of the scale, the number of items in the 'risks' component should be increased. Generally, fewer than three items of the factors are considered unstable, and the Cronbach $\alpha$ calculation has limitations for a two-item subscale [38]. The risks subscale of the scale should consist of 3 items. These differences were due to the adaptation of the scale [15-17]. Vaccine hesitancy is a complex and multidimensional issue [16, 25, 39]. Similarly, with other studies, [16, 32, 37]. our findings suggest that our adaptation is far from one-dimensional VHS. It showed that it makes it very two-dimensional because trust and risk structures have been recognized as part of vaccine hesitation.

The Cronbach alpha for the VHS total scale in the study was 0.80 . The Cronbach alpha for the first subdimension 'lack of confidence' was .90 and the second sub-dimension 'risks' was 0.74 . In the study by Shapiro et al. [16], Cronbach's was $\alpha 0.92$ for 'lack of confidence' and Cronbach's $\alpha 0.64$ for 'risks' . Domek et al. [15], Cronbach's alpha values for the confidence subscale and the peace of mind subscale were 0.78 and 0.70 , respectively. Likewise, studies noted that Cronbach alpha values were higher [15]. The Cronbach alpha coefficient, which is a measure of the internal consistency of items, is used to explain or question the homogeneous structure of the items in the scale. It is interpreted that the items in the scale with a high Cronbach alpha coefficient consist of items that are consistent with each other and that measure the same feature. If the Cronbach alpha coefficient is $0.60-0.80$, it is highly reliable, and $0.80-1.00$ indicates that it is highly reliable [36]. The data of this study were found to be reliable.

The retest reliability of VHS shows that it measures the relevant structure properly. Also, a large correlation between VHS subscales and retest indicates that VHS has criterion validity. The fact that the subscale means were almost the same for the study group and the retest group supports the accuracy of the study in terms of both validity and reliability. Likewise, the findings of the two data groups in the lack of confidence and risks subscale are very close to each other. The parallelism of the averages in the study also shows the consistency of the findings in two different periods and provides evidence about the validity and reliability of the scale.

Vaccine hesitancy is complex and specific to the situation and varies with time, place and types of the vaccine. It is affected by factors such as indifference, comfort and trust [13]. Vaccine hesitancy is constant and it can be measured by evaluating attitudes and beliefs towards infectious diseases. The multifactorial and complex causes of the hesitancy around vaccination require a wide variety of approaches, interventions and policy changes. These changes also should be implemented at the individual, community, health system and national levels. Improving understanding of how these factors vary between different subpopulations among healthcare providers, the healthcare system and public health authorities can help the studies of vaccine hesitancy. Importantly, this scale can help us understand the individuals' level of vaccine hesi- 
tancy during the COVID-19 epidemic as this understanding can develop appropriate interventions. Therefore, standardized measurement tools such as those proposed by SAGE will make it easier to measure the amount of hesitancy. It is necessary to have the capacity to measure the geographic clustering of hesitancy. Changes in the prevalence of hesitancy over time through serial, cross-sectional questionnaires using standardized questions and methods are critical [20]. The fact that the findings obtained as a result of statistical analysis are quite compatible with the data in the original article of VHS shows the validity and reliability of the scale [16].

\section{Limitations}

The findings of the study should be interpreted within the geographical, socioeconomic and sociocultural context of the research participants and areas. Retest data were obtained by contacting the people in the study group after an average of 2 weeks. It was very difficult to reach even 76 people out of 246 as a repeat group.

\section{CONCLUSION}

Minimizing vaccine hesitancy is an international priority. VHS has been adapted and used in many countries, but these countries are generally in the United States of America and Europe. While there might have been cultural differences regarding the Turkish form of the scale at the beginning of the study, the data showed that VHS is quite compatible with Turkish culture. VHS has been found two factors including 'lack of confidence' and 'risks' with structure and criterion validity in identifying individuals with vaccine hesitancy. Shapiro et al. [16] evaluated the reliability of the retest and found it to be reliable. A standardized, validated measure of vaccine hesitancy beliefs will help advance the research and vaccination policy.

\section{Authors' Contribution}

Study Conception: HYD, İ, ZT; Study Design: HYD, ID, ZT; Supervision: HYD; Funding: HYD, ID, ZT; Materials: HYD; Data Collection and/or Processing: HYD, ID, ZT; Statistical Analysis and/or Data Interpretation: HYD, İ; Literature Review: HYD, ID,
ZT; Manuscript Preparation: HYD, ID, ZT and Critical Review: HYD, İD, ZT.

\section{Conflict of interest}

The authors disclosed no conflict of interest during the preparation or publication of this manuscript.

\section{Financing}

The authors disclosed that they did not receive any grant during conduction or writing of this study.

\section{REFERENCES}

1. Haverkate M, D'Ancona F, Giambi C, Johansen K, Lopalco PL, Cozza V, et al. On behalf of the VENICE project gatekeepers and contact points. Mandatory and Recommended Vaccination in the EU, Iceland and Norway: Results of the VENICE 2010 Survey on the Ways of Implementing National Vaccination Programmes. Euro Surveill 2012;17:20183.

2. Bozkurt Bekis H. [An overview of vaccine rejection and review of literature]. Kafkas J Med Sci 2018;8:71-6. [Article in Turkish]

3. WHO. Immunization coverage. 2018; URL: https://www.who.int/en/news-room/fact-sheets/detail/immunization-coverage.

4. Poland GA, Jacobson RM. The age-old struggle against the antivaccinationists. New Engl J Med 2011;364:97-9.

5. Leask J. Should we do battle with antivaccination activists? Public Health Res Pract 2015;25:e2521515.

6. Callender D. Vaccine hesitancy: more than a movement. Hum Vaccin Immunother 2016;12:2464-8.

7. Dubé E, Gagnon D, Nickels E, Jeram S, Schuster M. Mapping vaccine hesitancy-Country-specific characteristics of a global phenomenon. Vaccine 2014;32:6649-54.

8. Dube E, Gagnon D, Ouakki M, Bettinger JA, Guay M, Halperin $\mathrm{S}$, et al. Understanding vaccine hesitancy in Canada: results of a consultation study by the Canadian immunization research network. PLoS One 2016;11:e0156118.

9. Roberts JR, Thompson D, Rogacki B, Hale JJ, Jacobson RM, Opel DJ, et al. Vaccine hesitancy among parents of adolescents and its association with vaccine uptake. Vaccine 2015;33:174855.

10. MacDonald N E. the SAGE Working Group on Vaccine Hesitancy. Vaccine hesitancy: definition, scope and determinants. Vaccine 2015;33:4161-4.

11. Larson HJ, Jarrett C, Eckersberger E, Smith DM, Paterson P. Understanding vaccine hesitancy around vaccines and vaccination from a global perspective: a systematic review of published literature, 2007-2012. Vaccine 2014;32:2150-9.

12. Shapiro GK, Perez S, Naz A, Tatar O, Guichon J, Amsel R, et al. Investigating Canadian parents' HPV vaccine knowledge, attitudes, and behaviour: a study protocol for a longitudinal national online survey. BMJ Open 2017;7:e017814

13. WHO, The SAGE Vaccine Hesitancy Working Group. What 
Influences Vaccine Acceptance: A Model of Determinants of Vaccine Hesitancy. Access date: 08.09.2020. https:/www.who.int/immunization/sage/meetings/2014/october/1_Report_WORKING_GROUP_vaccine_hesitancy_final.pd $\mathrm{f}$

14. Larson HJ, Jarrett C, Schulz WS, Chaudhuri M, Zhou Y, Dube E, et al. Measuring vaccine hesitancy: the development of a survey tool. Vaccine 2015;33:4165-75.

15. Domek GJ, O'Leary ST, Bull S, Bronsert M, ContrerasRoldan IL, Bolaños Ventura GA, et al. Measuring vaccine hesitancy: field testing the WHO SAGE Working Group on Vaccine Hesitancy survey tool in Guatemala. Vaccine 2018;36:5273-81. 16. Shapiro GK, Tatar O, Dube E, Amsel R, Knauper B, Naz A, et al. The vaccine hesitancy scale: psychometric properties and validation. Vaccine 2018;36:660-7.

17. Luyten J, Bruyneel L, van Hoek AJ. Assessing vaccine hesitancy in the UK population using a generalized vaccine hesitancy survey instrument. Vaccine 2019;37:2494-2501.

18. Sabahelzain MM, Dubé E, Moukhyer M, Larson HJ, van den Borne B, Bosma H. Psychometric properties of the adapted measles vaccine hesitancy scale in Sudan. PLoS One 2020;15:e0237171.

19. Bertin P, Nera K, Delouvée S. Conspiracy beliefs, rejection of vaccination, and support for hydroxychloroquine: a conceptual replication-extension in the COVID-19 pandemic context. Front Psychol 2020;11:565128.

20. Fadda M, Albanese E, Suggs LS. When a COVID-19 vaccine is ready, will we all be ready for it? Int J Public Health 2020;65:711-2.

21. Le TT, Andreadakis Z, Kumar A. The COVID-19 vaccine development landscape. Nat Rev Drug Discov 2020;19:305-6.

22. Magadmi RM, Fatemah OK. Beliefs and barriers associated with COVID-19 vaccination among the general population in Saudi Arabia. Reserach Square 2020;1-17.

23. Palamenghi L, Barello S, Boccia S, Graffigna G. Mistrust in biomedical research and vaccine hesitancy: the forefront challenge in the battle against COVID-19 in Italy. Eur J Epidemiol 2020;35: 785-8.

24. Gilkey MB, Reiter PL, Magnus BE, McRee AL, Dempsey AF, Brewer NT. Validation of the vaccination confidence scale: a brief measure to identify parents at risk for refusing adolescent vaccines. Acad Pediatr 2016;16:42-9.

25. Opel DJ, Taylor JA, Mangione-Smith R, Solomon C, Zhao C, Catz S, et al. Validity and reliability of a survey to identify vaccine-hesitant parents. Vaccine 2011;29:6598-605.

26. Shapiro GK, Holding A, Perez S, Amsel R, Rosberger Z. Validation of the vaccine conspiracy belief scale. Papillomavirus Res 2016;2:167-72.

27. Martin LR, Petrie KJ. Understanding the dimensions of antivaccination attitudes: the Vaccination Attitudes Examination (VAX) scale. Ann Behav Med 2017;51:652-60.

28. Horne Z, Powell D, Hummel JE, Holyoak KJ. Countering antivaccination attitudes. Proc Natl Acad Sci U S A 2015;112:10321-4.

29. Esin MN. Veri toplama yöntem ve araçları \& veri toplama araçlarının güvenirlik ve geçerliği. Erdoğan S, Nahcivan N, Esin MN (eds.), Hemşirelikte Araştırma: Süreç, Uygulama ve Kritik, İstanbul: Nobel Tıp Kitabevleri, 2014.

30. Karakoç FY, Dönmez L. [Basic principles of scale development]. Tıp Eğitimi Dünyası 2014;40:39-49. [Article in Turkish] 31. Lawshe $\mathrm{CH}$. A quantitative approach to content validity 1 . Personnel Psychology 1975;28:563-75.

32. Ayre C, Scally AJ. Critical values for Lawshe's content validity ratio: revisiting the original methods of calculation. Meas Eval Couns Dev 2014;47:79-86.

33. Raubenheimer J. An item selection procedure to maximise scale reliability andvalidity. SA J Industrial Psychology 2004;30;168-74.

34. Şencan H. Sosyal ve davranışsal ölçümlerde güvenilirlik ve geçerlik. Seçkin yayıncılık, Ankara, 2005.

35. Taşkın Ç, Akat Ö. Araştırma yöntemlerinde yapısal eşitlik modelleme. Ekin Yayınevi, Bursa. 2010.

36. Yıldız D, Uzunsakal E. [A comparison of reliability tests in field researches and an application on agricultural data]. Uygulamalı Sosyal Bilimler Dergisi 2018;1:16-21. [Article in Turkish] 37. Luyten J, Bruyneel L, van Hoek AJ. Assessing vaccine hesitancy in the UK population using a generalized vaccine hesitancy survey instrument. Vaccine 2019;37:2494-2501.

38. Eisinga R, te Grotenhuis M, Pelzer B. The reliability of a twoitem scale: Pearson, Cronbach, or Spearman-Brown? Int J Public Health 2013;58:637-42.

39. Peretti-Watel P, Larson HJ, Ward JK, Schulz WS, Verger P. Vaccine hesitancy: clarifying a theoretical framework for an ambiguous notion. PLoS Curr 2015;7: ecurrents.outbreaks.6844c80ff9f5b273f34c91f71b7fc289 40. Somon DA, Dudley MZ, Glanzc JM, Omer SB. Vaccine hesitancy: causes, consequences, and a call to action. Vaccine 2015;9(6 Suppl 4):391-8. 